\title{
Experiences with Inquiry-Based Learning in an Introductory Mechanics Course
}

\author{
Devin R. Berg \\ Engineering \& Technology Department \\ University of Wisconsin - Stout \\ 18 October 2013
}




\section{How do I relate the stuff in class to my everyday life?}
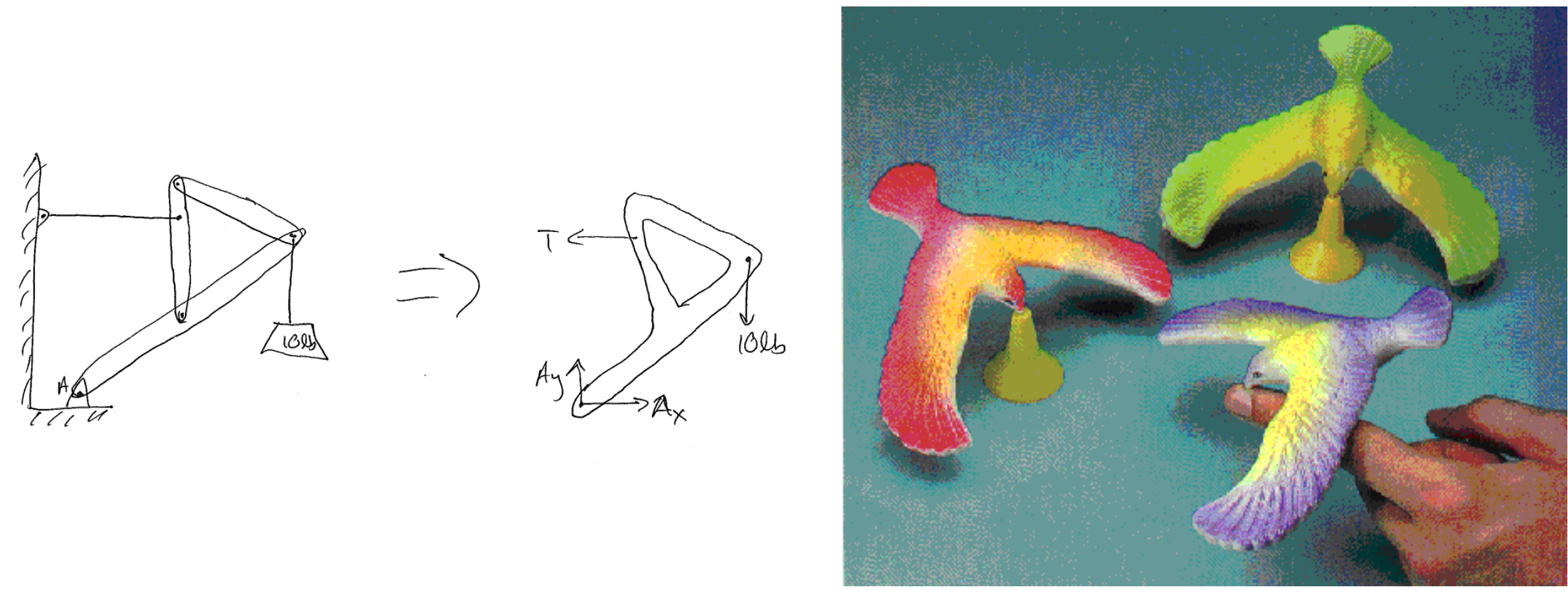

http://www.ux1.eiu.edu/ cfadd/1350/12Statics/ToC.html 


\section{Inquiry-based learning enables greater concept retention.}

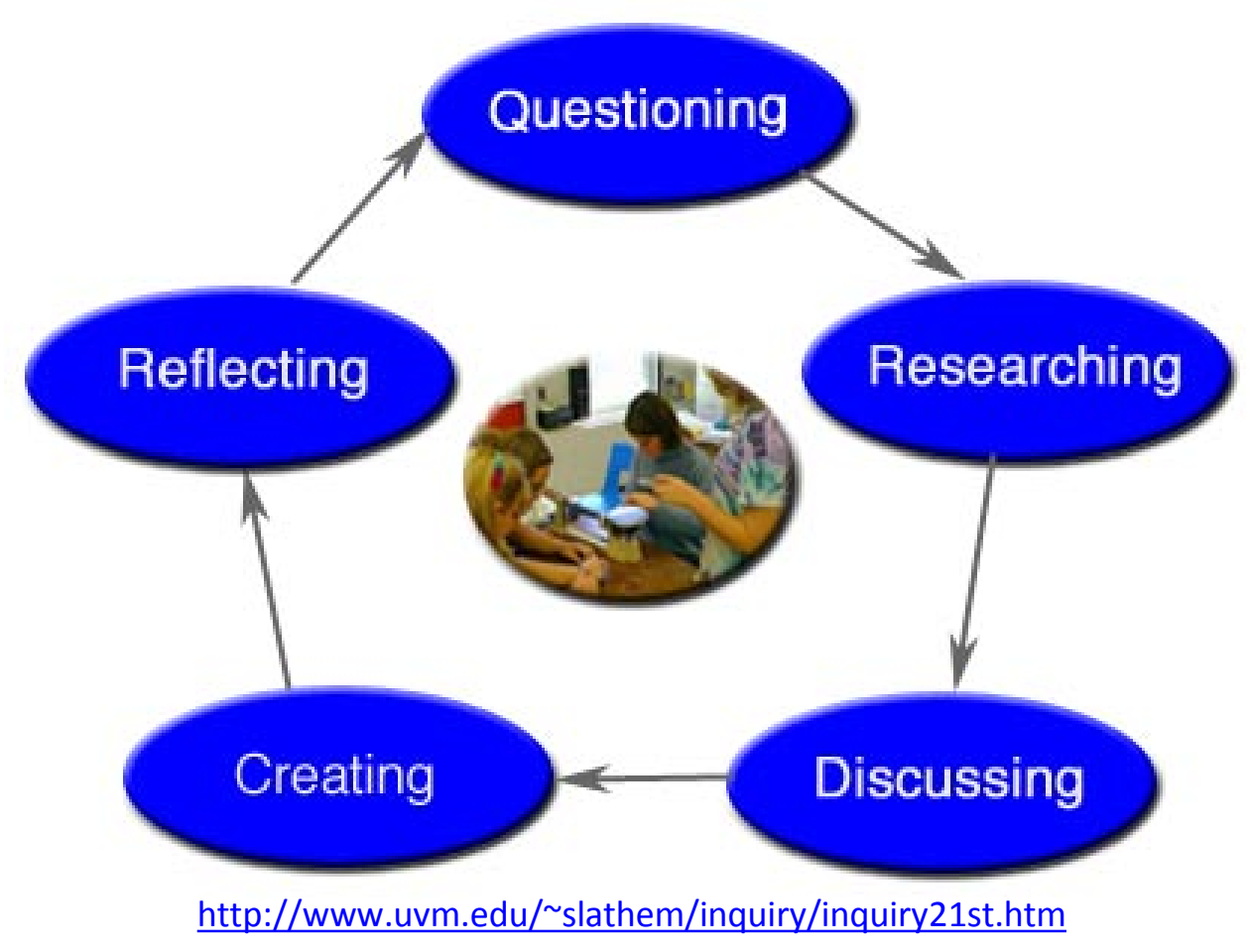

ENGINEERING AND TECHNOLOGY DEPARTMENT

Inspiring Innovation Learn more at www. uwstout.edu/et/ 


\section{Ask them to do this directly!}
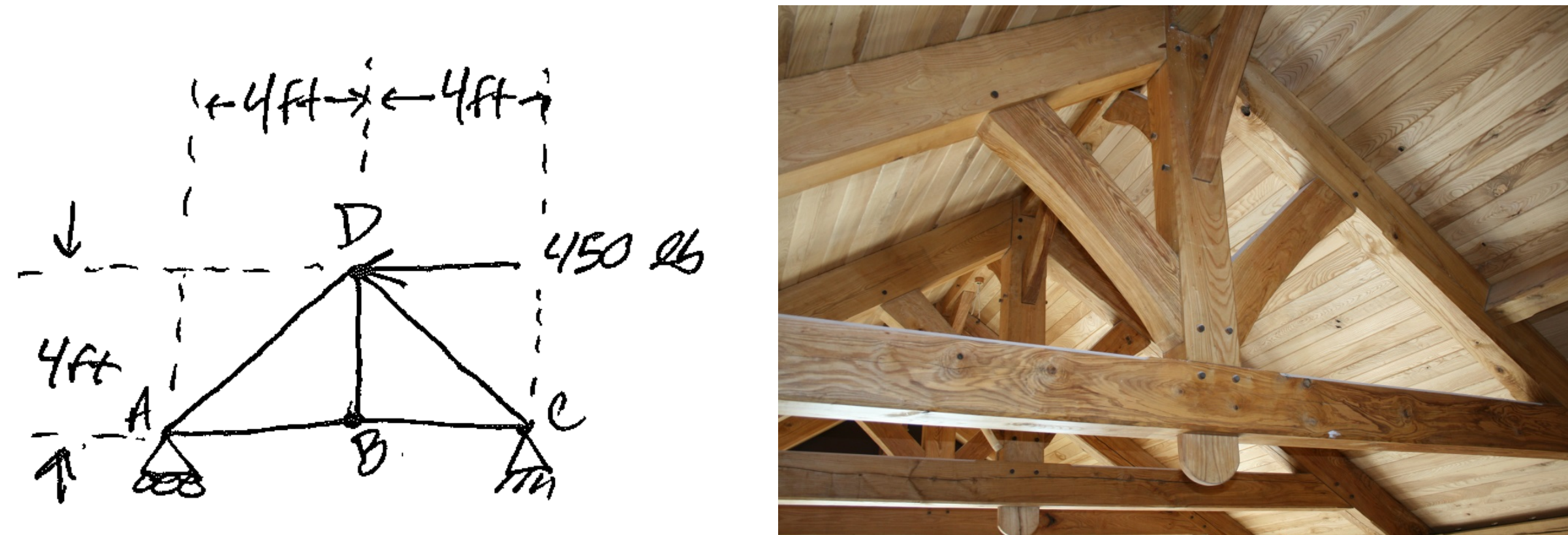

http://www.trilliumdell.com/timber-frame-trusses-and-accents.html 


\section{An Initial Pilot Study}

The goal of this study was to establish a baseline for student participation in an inquiry-based assignment.

The observations made here would then inform future efforts in this direction. 


\section{Basic instructions were given as a guide but were open to interpretation.}

Find an example of an object that demonstrates particle or rigid body equilibrium around campus. Capture a photograph of it. Write a one paragraph explanation of the photograph that includes at least an explanation of how many forces are acting on the object, whether you would need to perform 2D or 3D analysis of the object, and where you took the photograph. 


\section{$77 \%$ of submissions were "planar" and most had simple loading scenarios.}

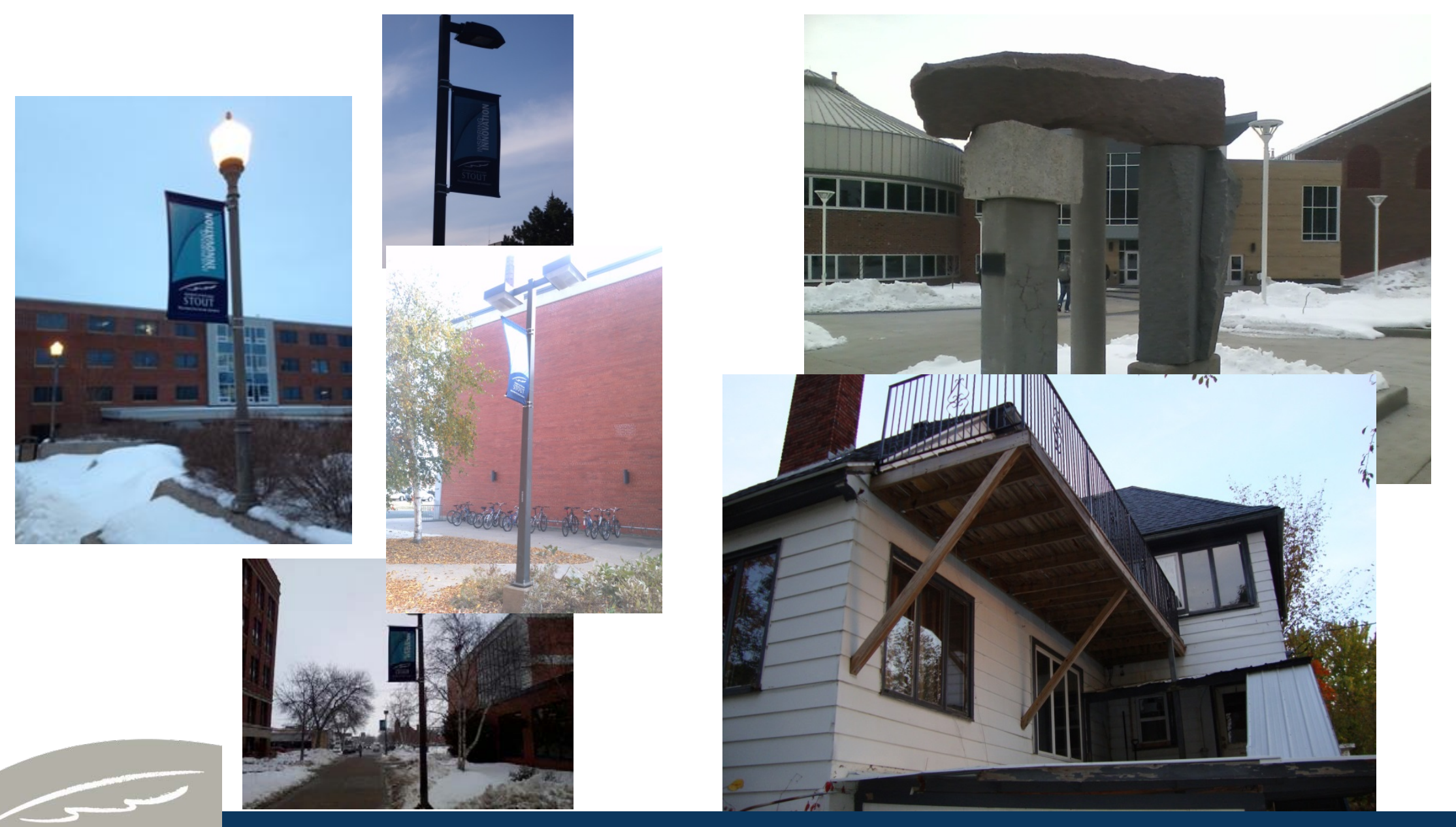




\section{$23 \%$ presented a more interesting example of static equilibrium.}
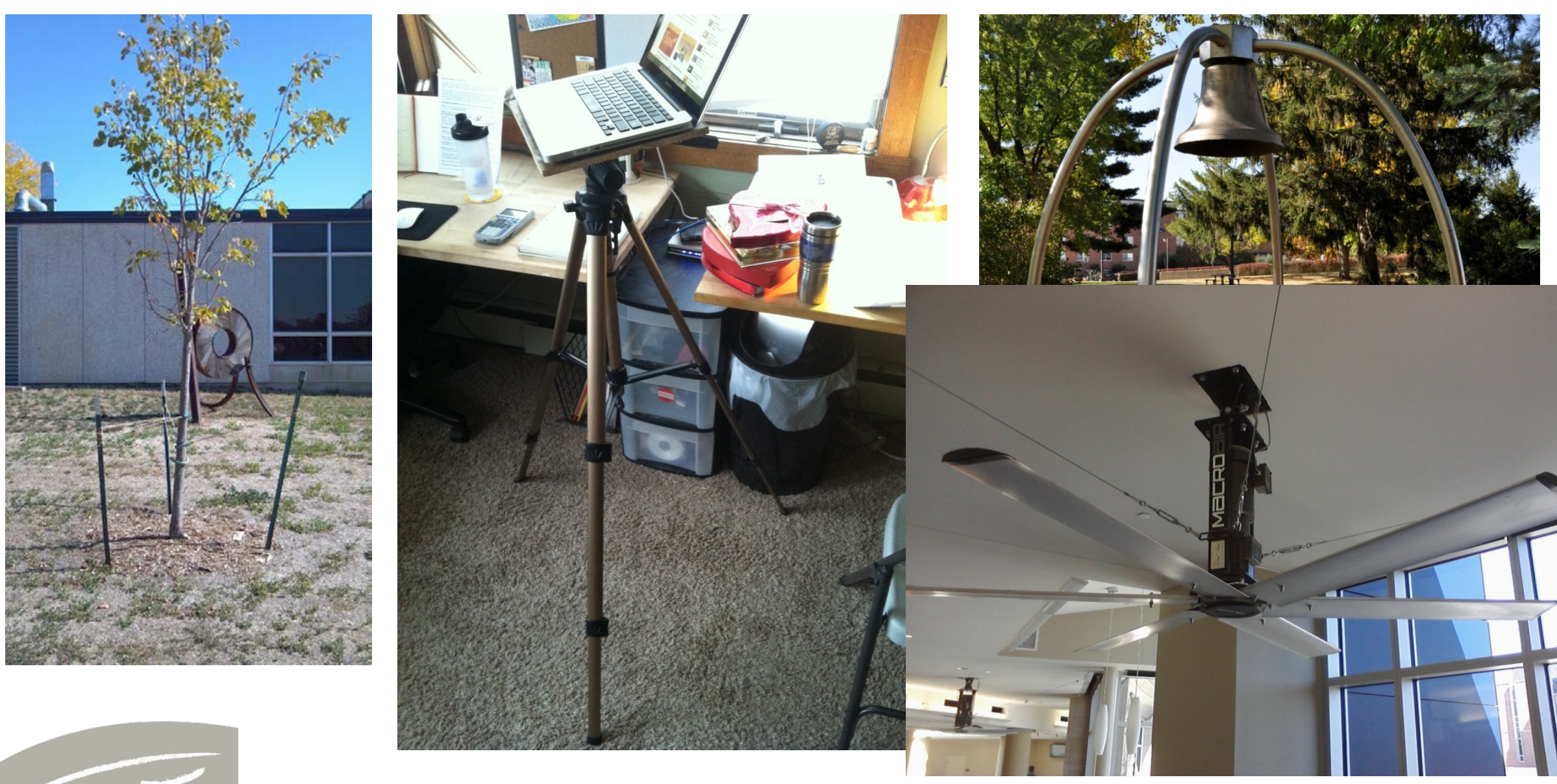

STOUT

ENGINEERING AND TECHNOLOGY DEPARTMENT

Inspiring Innovation Learn more at www.uwstout.edu/et/ 


\section{D submission were of higher quality when assessed for accuracy.}

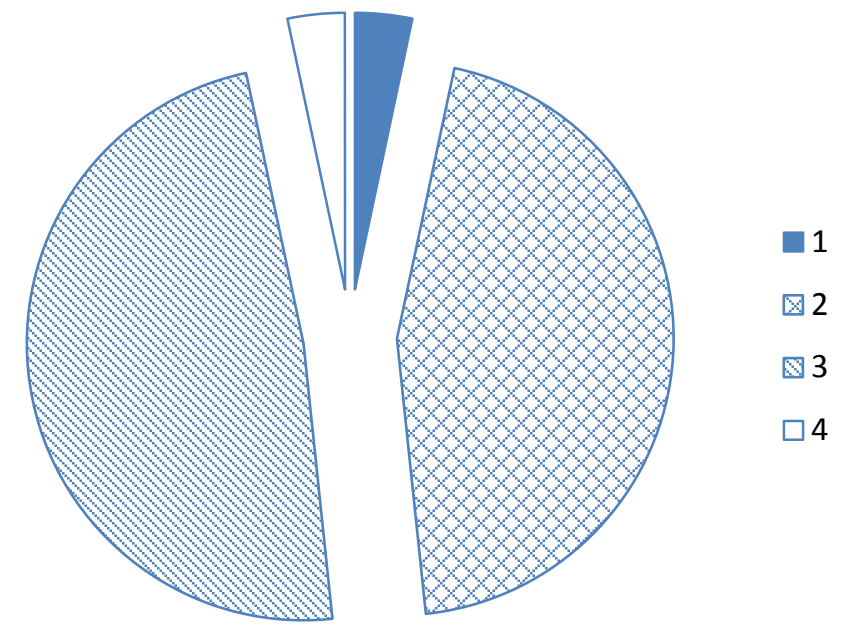

$52 \%$ of planar submissions scored 3 or 4

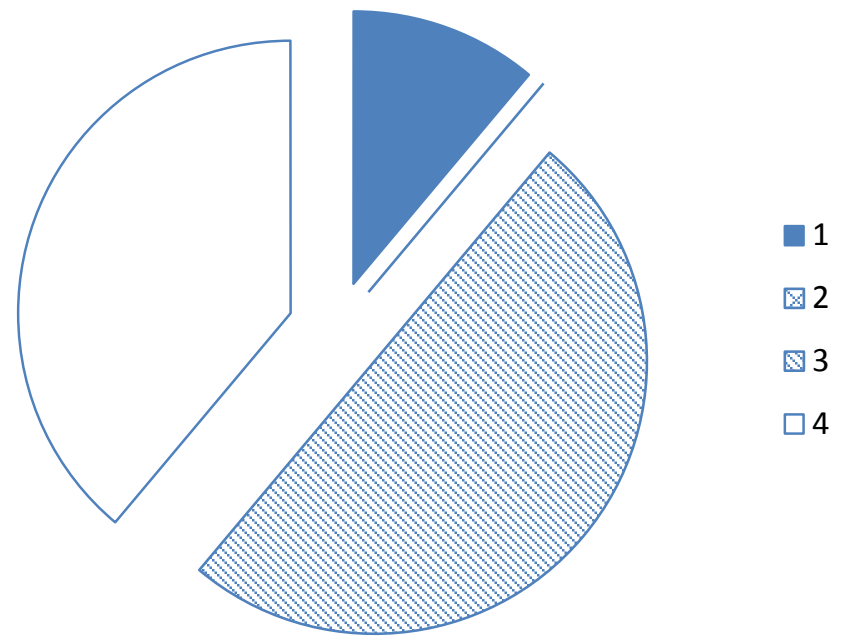

$89 \%$ of 3D submissions scored 3 or 4 


\section{Several students constructed a system under equilibrium to analyze.}

I determined that the center of mass of the book was hanging over the edge of the desk, and because gravity is creating a downward force the book should tilt and fall. There is also a moment balance, at the point where the edge of the desk and book meet. The moment is the force being applied in the downward direction (weight of the book) multiplied by the distance it is from the focal point. There was a second force that provided enough force to keep the body in equilibrium. This second force was the jar of pencils. The jar had a downward force on the book, however, had enough force to in the negative direction overcome the reaction on the book to keep it from tilting and falling. It was sufficient enough to analyze this object in 2D.

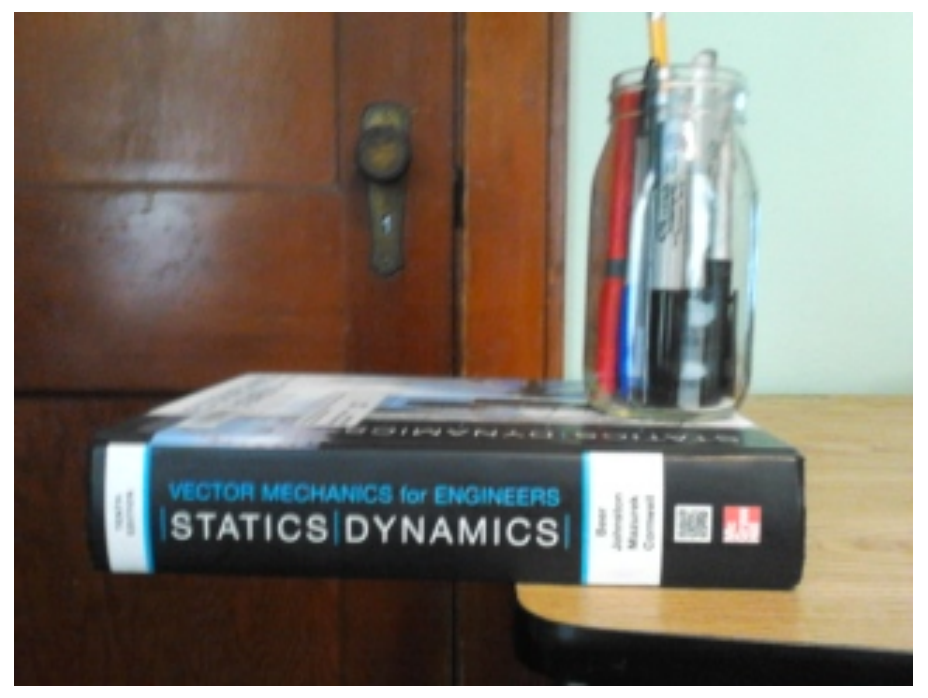




\section{The submissions collected support}

further use of this form of assignment.

Students: - enjoyed the assignment - required a different way of thinking

Instructor: - helpful when gaging student understanding - more engaging discussion 


\section{Currently using this form of assignment on weekly basis.}

Students have started to tell me about things they saw that relate to what they have learned.

Some have even begun collecting evidence of different examples before being assigned to do so. 


\section{Acknowledgements}

The author would like to thank the UW-Stout students in the Fall 2012 and Spring 2013 semester sections of MECH 290 and MECH 293 for their photographic and textual contributions. 


\section{Thank you for your time!}
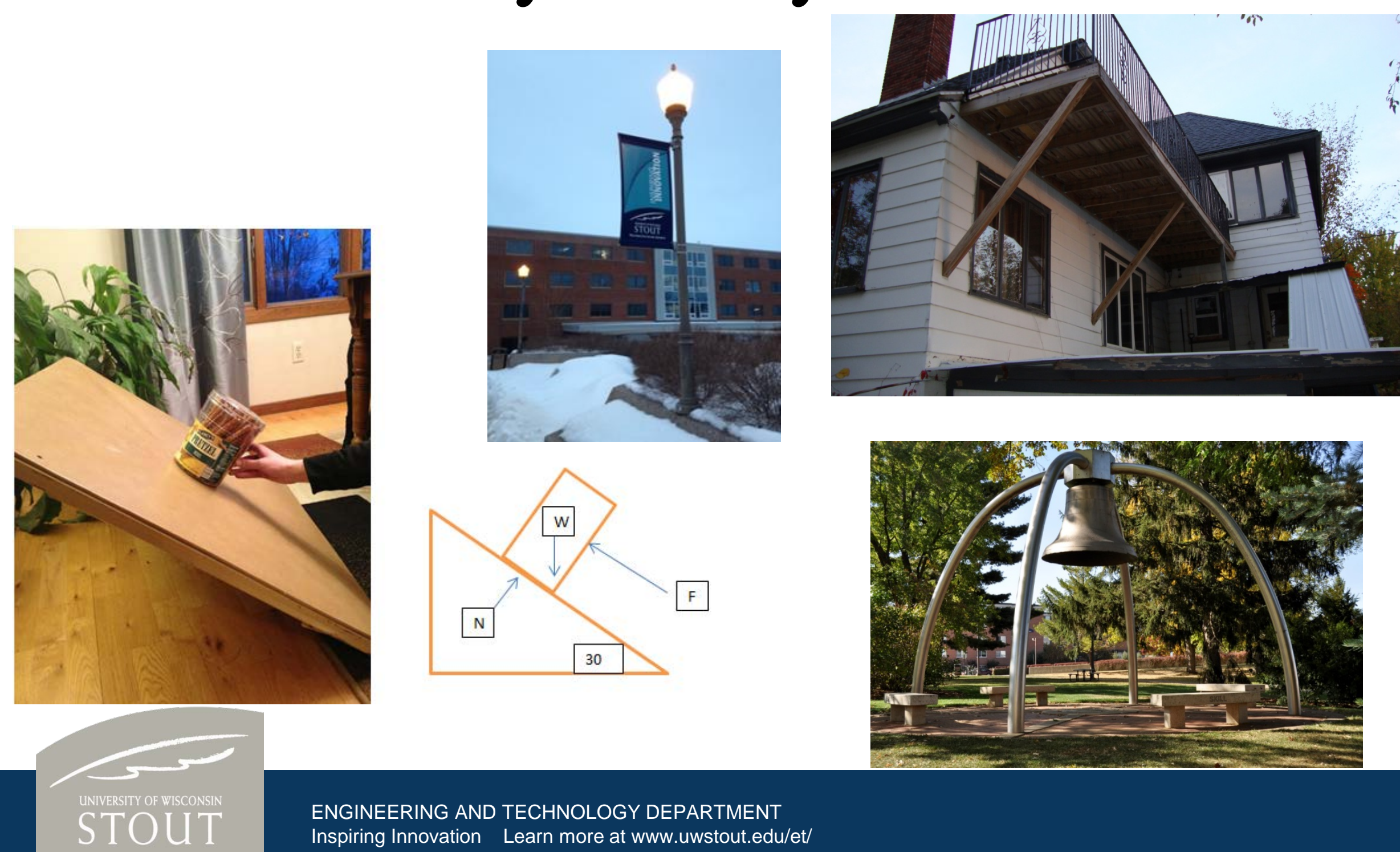

ENGINEERING AND TECHNOLOGY DEPARTMENT

Inspiring Innovation Learn more at www.uwstout.edu/et/ 